\title{
Shear Driven Micro-Flows of Gaseous Mixtures
}

\author{
Stergios Naris and Dimitris Valougeorgis* \\ Department of Mechanical and Industrial Engineering, University of Thessaly, Pedion Areos, Volos 38334, Greece
}

(Received: 2 February 2005. Accepted: 30 September 2005)

\begin{abstract}
A mesoscale kinetic-type approach is proposed to solve shear driven micro flows of binary gas mixtures in MEMS. The coupled linear integro-differential equations, which formally describe the flow, are solved using the discrete velocity method. The complicated collision integral term is approximated by the McCormack model. The proposed approach is applied in one and two dimensions, solving the Couette and the driven cavity problems respectively, for two binary gas mixtures ( $\mathrm{Ne}-\mathrm{Ar}$ and $\mathrm{He}-\mathrm{Xe}$ ). Numerical results are presented for a wide range of the rarefaction and for various molar concentrations. It is demonstrated that the formulation is very efficient and can be implemented as an alternative to classical approaches, such as Navier Stokes solvers with slip boundary conditions.
\end{abstract}

\section{Keywords:}

\section{INTRODUCTION}

Fluid mechanics and transport phenomena at microscale level are very important in the detailed design of microelectromechanical devices and equipment. ${ }^{1,2}$ In most cases these problems have been studied by implementing the continuum conservation equations, supplemented by the appropriate slip boundary conditions.

It is argued however, that for various reasons related to the physics as well as to the numerics involved, an alternative promising approach is to treat the problem at the mesoscopic level via a kinetic type scheme. ${ }^{3-5}$ For example, it is evident that a solution at the macroscopic level is valid only for moderate gas rarefaction. Also, the accurate estimation of the slip coeffients, which are required for the efficient implementation of the slip boundary conditions depend on the solution of the Boltzmann equation. In addition, in many occasions, simulation of gas micro flows in MEMS components require a coupling of a DSMC code with a Navier Stokes solver. This is a complicated task, which can be avoided when a mesoscale approach is followed. Finally recent developments in computational kinetic theory facilitates the efficient solution of even multi-dimensional flow configurations based on kinetic equations. ${ }^{6}$

Gaseous flows of a single gas in the whole range of rarefaction, has been extensively studied over the years and a number of interesting phenomena, which vanish at the continuum limit, have been revealed. ${ }^{7}$ In spite of the

${ }^{*}$ Corresponding author; E-mail: diva@mie.uth.gr great practical importance, the corresponding gaseous mixture rarefied flows have received much less attention. ${ }^{8}$ The main problem is that the computational effort is significantly increased when we move from a single gas to a gas mixture. Moreover, in mixtures new phenomena arise due to the concentration gradient. Recently, the case of a binary gas mixture flow in half space and in slab and axisymmetric geometry has been examined, based on the McCormack model, ${ }^{9}$ in a more detailed and systematic manner. ${ }^{10-13}$ In addition, the flow of gaseous mixtures in a 2-D rectangular duct due to a pressure gradient in the longitudinal direction has been solved. ${ }^{14,15}$

Here, this latter work with the McCormack model is modified accordingly to study shear driven flows of binary gas mixtures. The proposed methodology is demostrated by solving in one and two dimensions, the Couette and driven cavity problems respectively. Existing work for these two prototype problems is very limited. The binary Couette flow problem has been solved in Refs. [16-18], by using the Hamel mode ${ }^{19}$ and very recently by applying the McCormack model. ${ }^{20}$ The micro cavity problem has been studied only for a single gas, ${ }^{2,21,22}$ while for a binary gas mixture, as far as the authors are aware of, there are no available results in the literature.

\section{FORMULATION}

The molar concentration of each species in the mixture is

$$
C_{\alpha}=\frac{n_{0 \alpha}}{n_{01}+n_{02}}, \quad \alpha=1,2
$$


where $n_{0 \alpha}(\alpha=1,2)$ is a reference number density of species $\alpha$ and $C_{1}+C_{2}=1$. Here, we choose to denote $C_{1}=C$ and consequently $C_{2}=1-C$. The mean molecular mass of the mixture is defined as

$$
m=C m_{1}+(1-C) m_{2}
$$

where $m_{\alpha}$ is the molecular mass of species $\alpha$. The rarefaction parameter is given by

$$
\delta=\frac{H P_{0}}{\mu}\left(\frac{m}{2 k T_{0}}\right)^{1 / 2}
$$

where $H$ is the characteristic length of the problem, $P_{0}$ is a reference pressure, $\mu$ is the viscosity of the mixture at temperature $T_{0}$ and $k$ is the Boltzmann constant. Considering that the viscosity is proportional to the molecular mean free path $\lambda$, one can see that the rarefaction parameter $\delta$ is proportional to the inverse Knudsen number defined as $K n=\lambda / H$.

The linearized form of the model equations, proposed by $\mathrm{McCormack}^{9}$ to describe the stationary state of a binary gas mixture is

$$
\mathbf{c}_{\alpha} \cdot \nabla \Phi_{\alpha}=\sum_{\beta=1}^{2} L_{\alpha \beta} \Phi_{\alpha} \quad \alpha=1,2
$$

where $\Phi_{\alpha}\left(\mathbf{r}, \mathbf{c}_{\alpha}\right)$ are the unknown perturbation functions, $\mathbf{r}=(x, y, z)$ and $\mathbf{c}_{\alpha}=\left(c_{x}, c_{y}, c_{z}\right)$ are the dimensionless position vector and molecular velocity respectively, while the linearized collision operator is given by

$$
\begin{aligned}
L_{\alpha \beta}\left(\mathbf{c}_{\alpha}\right)= & -\gamma_{\alpha \beta} \Phi_{\alpha}+\gamma_{\alpha \beta} \rho_{\alpha} \\
& +2\left\{\gamma_{\alpha \beta} u_{\alpha i}-\left[u_{\alpha i}-\left(\frac{m_{\alpha}}{m_{\beta}}\right)^{1 / 2} u_{\beta i}\right] \nu_{\alpha \beta}^{(1)}\right. \\
& \left.-\left[q_{\alpha i}-\left(\frac{m_{\alpha}}{m_{\beta}}\right)^{3 / 2} q_{\beta i}\right] \nu_{\alpha \beta}^{(2)}\right\} c_{\alpha i} \\
+ & {\left[\gamma_{\alpha \beta} T_{\alpha}-2 \frac{m_{\alpha \beta}}{m_{\beta}}\left(T_{\alpha}-T_{\beta}\right) \nu_{\alpha \beta}^{(1)}\right]\left(c_{\alpha}^{2}-\frac{3}{2}\right) } \\
+ & 2\left[\left(\gamma_{\alpha \beta}-\nu_{\alpha \beta}^{(3)}\right) \Pi_{\alpha i j}+\nu_{\alpha \beta}^{(4)} \Pi_{\beta i j}\right] c_{\alpha i} c_{\alpha j} \\
+ & \frac{8}{5}\left\{\left(\gamma_{\alpha \beta}-\nu_{\alpha \beta}^{(5)}\right) q_{\alpha i}+\nu_{\alpha \beta}^{(6)} q_{\beta i}\right. \\
& \left.\quad-\frac{5}{8}\left[u_{\alpha i}-\left(\frac{m_{\alpha}}{m_{\beta}}\right)^{1 / 2} u_{\beta i}\right] \nu_{\alpha \beta}^{(2)}\right\} c_{\alpha i}\left(c_{\alpha}^{2}-\frac{5}{2}\right)
\end{aligned}
$$

Closed form expressions for the collision frequencies $\gamma_{\alpha \beta}$ and the parameters $\nu_{\alpha \beta}$ in Eq. (5), are given in Refs. $[9,10]$. The quantities $\rho_{\alpha}, u_{\alpha i}, q_{\alpha i}, T_{\alpha}$, and $\Pi_{\alpha i j}$ are the dimensionless departures of density, hydrodynamic velocity, heat flux, temperature and stresses of the species $\alpha$ from their corresponding reference values. Finally the subscripts $i, j=x, y, z$ denote repeated tensor indices.
For the particular problems under consideration, $\Phi_{\alpha}$ is a function only of two spatial variables, say $x$ and $y$, while the temperature perturbation is assumed equal to zero. In addition, the $z$-component of the molecular velocity, can be eliminated by introducing the reduced distribution functions

$$
h_{\alpha}\left(x, y, c_{\alpha x}, c_{\alpha y}\right)=\frac{1}{\sqrt{\pi}} \int_{-\infty}^{\infty} \Phi_{\alpha} e^{-c_{\alpha z}^{2}} d c_{\alpha z}
$$

and

$$
\theta_{\alpha}\left(x, y, c_{\alpha x}, c_{\alpha y}\right)=\frac{1}{\sqrt{\pi}} \int_{-\infty}^{\infty} \Phi_{\alpha}\left(c_{\alpha z}^{2}-\frac{1}{2}\right) e^{-c_{\alpha z}^{2}} d c_{\alpha z}
$$

Then operating accordingly on Eq. (5) the following two kinetic equations are obtained:

$$
\begin{aligned}
c_{\alpha x} \frac{\partial h_{\alpha}}{\partial x}+c_{\alpha y} \frac{\partial h_{\alpha}}{\partial y}+d_{\alpha} \gamma_{\alpha} h_{\alpha} \\
=\sqrt{\frac{m_{\alpha}}{m}} d_{\alpha}\left\{\gamma_{\alpha} \rho_{\alpha}+2\left[\gamma_{\alpha} u_{\alpha i}-\left(u_{\alpha i}-u_{\beta i}\right) \nu_{\alpha \beta}^{(1)}\right.\right. \\
\left.-\left(q_{\alpha i}-\frac{m_{\alpha}}{m_{\beta}} q_{\beta i}\right) \nu_{\alpha \beta}^{(2)}\right] c_{\alpha i}+\left[\left(\gamma_{\alpha}-\nu_{\alpha \alpha}^{(3)}\right.\right. \\
\left.\left.+\nu_{\alpha \alpha}^{(4)}-\nu_{\alpha \beta}^{(3)}\right) \Pi_{\alpha i i}+\nu_{\alpha \beta}^{(4)} \sqrt{\frac{m_{\beta}}{m_{\alpha}}} \Pi_{\beta i i}\right] c_{\alpha i}^{2} \\
+\left[\left(\gamma_{\alpha}-\nu_{\alpha \alpha}^{(3)}+\nu_{\alpha \alpha}^{(4)}-\nu_{\alpha \beta}^{(3)}\right) \Pi_{\alpha z z}\right. \\
\left.+\nu_{\alpha \beta}^{(4)} \sqrt{\frac{m_{\beta}}{m_{\alpha}}} \Pi_{\beta z z}\right]+4\left[\left(\gamma_{\alpha}-\nu_{\alpha \alpha}^{(3)}+\nu_{\alpha \alpha}^{(4)}\right.\right. \\
\\
\left.\left.-\nu_{\alpha \beta}^{(3)}\right) \Pi_{\alpha x y}+\nu_{\alpha \beta}^{(4)} \sqrt{\frac{m_{\beta}}{m_{\alpha}}} \Pi_{\beta x y}\right] c_{\alpha x} c_{\alpha y} \\
+\frac{8}{5}\left[\left(\gamma_{\alpha}-\nu_{\alpha \alpha}^{(5)}+\nu_{\alpha \alpha}^{(6)}-\nu_{\alpha \beta}^{(5)}\right) q_{\alpha i}\right. \\
\left.+\nu_{\alpha \beta}^{(6)} \sqrt{\frac{m_{\beta}}{m_{\alpha}}} q_{\beta i}-\frac{5}{8}\left(u_{\alpha i}-u_{\beta i}\right) \nu_{\alpha \beta}^{(2)}\right] \\
\left.\quad c_{\alpha i}\left(c_{\alpha x}^{2}+c_{\alpha y}^{2}-2\right)\right\} \\
+(8) \\
+
\end{aligned}
$$

and

$$
\begin{gathered}
c_{\alpha x} \frac{\partial \theta_{\alpha}}{\partial x}+c_{\alpha y} \frac{\partial \theta_{\alpha}}{\partial y}+d_{\alpha} \gamma_{\alpha} \theta_{\alpha} \\
=d_{\alpha} \sqrt{\frac{m_{\alpha}}{m}}\left\{\left(\gamma_{\alpha}-\nu_{\alpha \alpha}^{(3)}+\nu_{\alpha \alpha}^{(4)}-\nu_{\alpha \beta}^{(3)}\right) \Pi_{\alpha z z}\right. \\
+\nu_{\alpha \beta}^{(4)} \sqrt{\frac{m_{\beta}}{m_{\alpha}}} \Pi_{\beta z z}+\frac{8}{10}\left[\left(\gamma_{\alpha}-\nu_{\alpha \alpha}^{(5)}+\nu_{\alpha \alpha}^{(6)}\right.\right. \\
\left.-\nu_{\alpha \beta}^{(5)}\right) q_{\alpha i}+\nu_{\alpha \beta}^{(6)} \sqrt{\frac{m_{\beta}}{m_{\alpha}}} q_{\beta i} \\
\left.\left.-\frac{5}{8}\left(u_{\alpha i}-u_{\beta i}\right) \nu_{\alpha \beta}^{(2)}\right] c_{\alpha i}\right\}
\end{gathered}
$$


In Eqs. $(8,9), \alpha, \beta=1,2$ with $\alpha \neq \beta, \gamma_{\alpha}=\gamma_{\alpha \alpha}+\gamma_{\alpha \beta}$, $i=x, y$,

$$
d_{\alpha}=\delta\left(\frac{C}{\gamma_{\alpha}}+\frac{1-C}{\gamma_{\beta}}\right) \sqrt{\frac{m_{\alpha}}{m}}
$$

and the macroscopic quantities are given by moments of the reduced distribution functions according to

$$
\begin{gathered}
\rho_{\alpha}=\frac{1}{\pi} \sqrt{\frac{m}{m_{\alpha}}} \int_{-\infty}^{\infty} \int_{-\infty}^{\infty} h_{\alpha} e^{-c_{\alpha x}^{2}-c_{\alpha y}^{2}} d c_{\alpha x} d c_{\alpha y} \\
u_{\alpha i}=\frac{1}{\pi} \sqrt{\frac{m}{m_{\alpha}}} \int_{-\infty}^{\infty} \int_{-\infty}^{\infty} c_{\alpha i} h_{\alpha} e^{-c_{\alpha x}^{2}-c_{\alpha y}^{2}} d c_{\alpha x} d c_{\alpha y} \\
q_{\alpha i}=\frac{1}{\pi} \sqrt{\frac{m}{m_{\alpha}}} \frac{1}{2} \int_{-\infty}^{\infty} \int_{-\infty}^{\infty} c_{\alpha i}\left[\left(c_{\alpha x}^{2}+c_{\alpha y}^{2}-2\right) h_{\alpha}+\theta_{\alpha}\right] \\
\times e^{-c_{\alpha x}^{2}-c_{\alpha y}^{2}} d c_{\alpha x} d c_{\alpha y} \\
\Pi_{\alpha i i}=\frac{1}{\pi} \sqrt{\frac{m}{m_{\alpha}}} \int_{-\infty}^{\infty} \int_{-\infty}^{\infty} \frac{1}{3}\left[\left(2 c_{\alpha i}^{2}-c_{\alpha j}^{2}-\frac{1}{2}\right) h_{\alpha}-\theta_{\alpha}\right] \\
\times e^{-c_{\alpha x}^{2}-c_{\alpha y}^{2}} d c_{\alpha x} d c_{\alpha y}, \quad i=x, y, \quad j=x, y, \quad i \neq j \\
\Pi_{\alpha z z}=\frac{1}{\pi} \sqrt{\frac{m}{m_{\alpha}}} \int_{-\infty}^{\infty} \int_{-\infty}^{\infty} \frac{1}{3}\left[-\left(c_{\alpha x}^{2}+c_{\alpha y}^{2}-1\right) h_{\alpha}+2 \theta_{\alpha}\right] \\
e^{-c_{\alpha x}^{2}-c_{\alpha y}^{2}} d c_{\alpha x} d c_{\alpha y}
\end{gathered}
$$

and

$$
\Pi_{\alpha x y}=\frac{1}{\pi} \sqrt{\frac{m}{m_{\alpha}}} \int_{-\infty}^{\infty} \int_{-\infty}^{\infty} c_{\alpha x} c_{\alpha y} h_{\alpha} e^{-c_{\alpha x}^{2}-c_{\alpha y}^{2}} d c_{\alpha x} d c_{\alpha y}
$$

In the present work both the Couette and the cavity flow problems are solved using Eqs. $(8,9)$ coupled with Eqs. (11-15) and the corresponding boundary conditions. For the Couette flow problem, since the flow is considered fully developed and one-dimensional, periodic boundary conditions are implemented in the $x$-direction, which is taken parallel to the plates. In the transverse direction Maxwell diffuse boundary conditions are considered, resulting to

$$
\begin{aligned}
h_{\alpha}\left(x, \frac{-1}{2}, c_{\alpha x}, c_{\alpha y}\right) & =\sqrt{\frac{m_{\alpha}}{m}} \rho_{\alpha}\left(x, \frac{-1}{2}\right)-c_{\alpha x} \quad c_{\alpha y}>0 \\
h_{\alpha}\left(x, \frac{1}{2}, c_{\alpha x}, c_{\alpha y}\right) & =\sqrt{\frac{m_{\alpha}}{m}} \rho_{\alpha}\left(x, \frac{1}{2}\right)+c_{\alpha x} \quad c_{\alpha y}<0
\end{aligned}
$$

and

$$
\theta_{\alpha}\left(x, \frac{-1}{2}, c_{\alpha x}, c_{\alpha y}\right)=\theta_{\alpha}\left(x, \frac{1}{2}, c_{\alpha x}, c_{\alpha y}\right)=0
$$

In the square cavity flow problem, the reduced distributions functions at the stationary and moving walls are:

$$
\begin{gathered}
h_{\alpha}\left(x, 0, c_{\alpha x}, c_{\alpha y}\right)=\sqrt{\frac{m_{\alpha}}{m}} \rho_{\alpha}(x, 0), \\
\theta_{\alpha}\left(x, 0, c_{\alpha x}, c_{\alpha y}\right)=0 \quad c_{\alpha y}>0 \\
h_{\alpha}\left(0, y, c_{\alpha x}, c_{\alpha y}\right)=\sqrt{\frac{m_{\alpha}}{m}} \rho_{\alpha}(0, y), \\
\theta_{\alpha}\left(0, y, c_{\alpha x}, c_{\alpha y}\right)=0 \quad c_{\alpha x}>0 \\
h_{\alpha}\left(1, y, c_{\alpha x}, c_{\alpha y}\right)=\sqrt{\frac{m_{\alpha}}{m}} \rho_{\alpha}(H, y), \\
\theta_{\alpha}\left(1, y, c_{\alpha x}, c_{\alpha y}\right)=0 \quad c_{\alpha x}<0 \\
h_{\alpha}\left(x, 1, c_{\alpha x}, c_{\alpha y}\right)=\sqrt{\frac{m_{\alpha}}{m}} \rho_{\alpha}(x, H)+2 c_{\alpha x}, \\
\theta_{\alpha}\left(x, 1, c_{\alpha x}, c_{\alpha y}\right)=0 \quad c_{\alpha y}>0
\end{gathered}
$$

In the present work, the macroscopic quantities under investigation include the mean velocity and the stress tensor of the mixture given by

$$
u(x, y)=C u_{1}(x, y)+(1-C) u_{2}(x, y)
$$

and

$$
\Pi(x, y)=C \sqrt{\frac{m_{1}}{m}} \Pi_{1 x y}(x, y)+(1-C) \sqrt{\frac{m_{2}}{m}} \Pi_{2 x y}(x, y)
$$

respectively.

\section{NUMERICAL SCHEME}

Equations $(8,9)$ are discretized first in the velocity space $\left(c_{\alpha x}, c_{\alpha y}\right)$ and then in the physical space $(x, y)$. A Gauss integration scheme of order $N$ is chosen to define the set of discrete velocities for each component of the particle velocity yielding a total number of $N^{2}$ discrete velocities. The discretization in the physical space is performed using the so-called diamond-difference scheme, which is a second order scheme. ${ }^{23}$ The overall quantities at the right hand side of Eqs. $(8,9)$, are estimated from Eqs. (11-15), using Gauss quadrature. The iterative solution of the discretized version of Eqs. $(8,9)$ is known as the discrete velocity method (DVM) and it is consisting of the following steps: (i) Assume $\rho_{\alpha}, u_{\alpha i}, q_{\alpha i}, \Pi_{\alpha x x}, \Pi_{\alpha y y}, \Pi_{\alpha z z}$, and $\Pi_{\alpha x y}$ and compute $h_{\alpha}$ and $\theta_{\alpha}$ from Eqs. $(8,9)$, (ii) Estimate the new values of $\rho_{\alpha}, u_{\alpha i}, q_{\alpha i}, \Pi_{\alpha x x}, \Pi_{\alpha y y}, \Pi_{\alpha z z}$, and $\Pi_{\alpha x y}$ from Eqs. (11-15), and (iii) Go back to step (i) and use the updated values of $\rho_{\alpha}, u_{\alpha i}, q_{\alpha i}, \Pi_{\alpha x x}, \Pi_{\alpha y y}, \Pi_{\alpha z z}$, and $\Pi_{\alpha x y}$ to compute the updated $h_{\alpha}$ and $\theta_{\alpha}$. The iterative procedure is ended when the imposed convergence criterion on the overall quantities is satisfied.

\section{RESULTS AND DISCUSSION}

The calculations have been carried out over a wide range of the rarefied parameter $\delta$. The gas mixtures of $\mathrm{Ne}-\mathrm{Ar}$ 


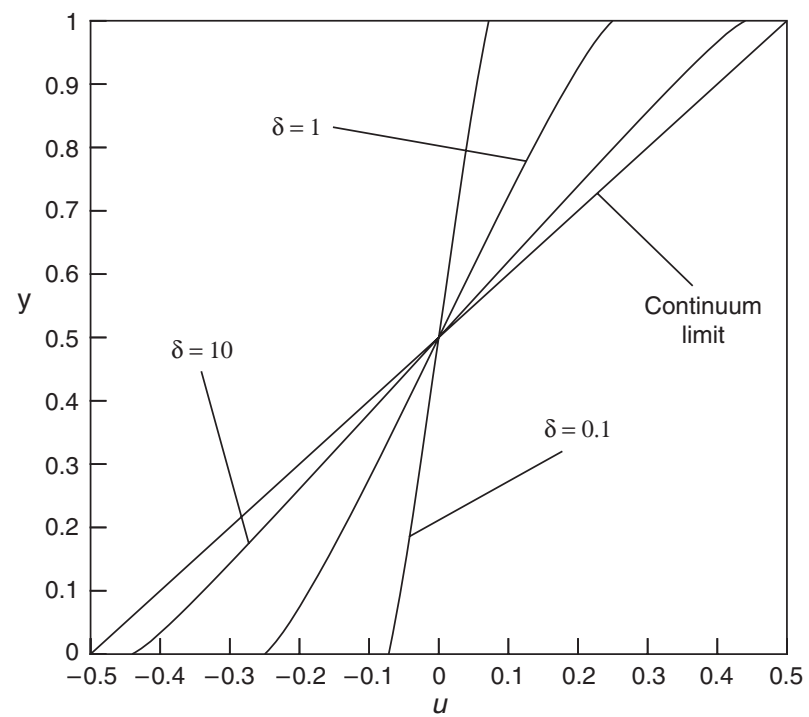

Fig. 1. Velocity profiles of the Couette flow, for $\mathrm{Ne}-\mathrm{Ar}(C=0.5)$ and various values of $\delta$.

and $\mathrm{He}-\mathrm{Xe}$ have been examined with molar concentrations $C=0.1,0.5$, and 0.9. The molecular masses are $m_{\mathrm{Ne}}=$ $20.1797, m_{\mathrm{Ar}}=39.948, m_{\mathrm{He}}=4.0026, m_{\mathrm{Xe}}=131.29$ in atomic units. Thus the study includes one mixture with particles of about equal masses and another one with particles of very different masses. The parameters $\nu_{\alpha \beta}$ have been calculated using the expressions given in Ref. [24] at temperature $T=300 \mathrm{~K}$, which have been obtained based on experimental data for the transport coefficients.

The presented non-dimensional results are based on a 64-point Gauss quadrature scheme to simulate the velocity space. The relative convergence criterion of the iteration scheme is $10^{-6}$.

First the results of the Couette flow problem are presented. In Figures 1 and 2, the mean velocity profiles are

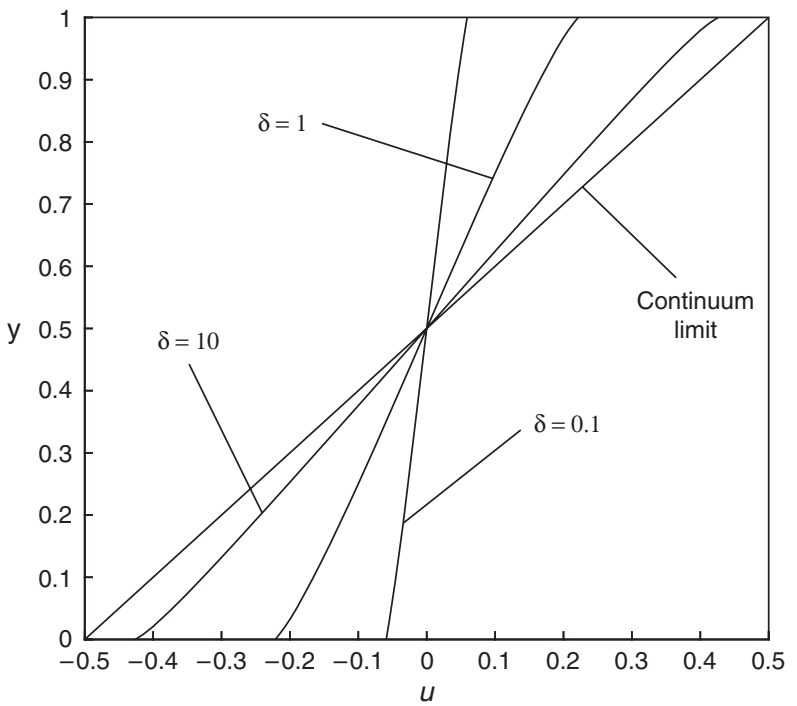

Fig. 2. Velocity profiles of the Couette flow, for $\mathrm{He}-\mathrm{Xe}(C=0.5)$ and various values of $\delta$.

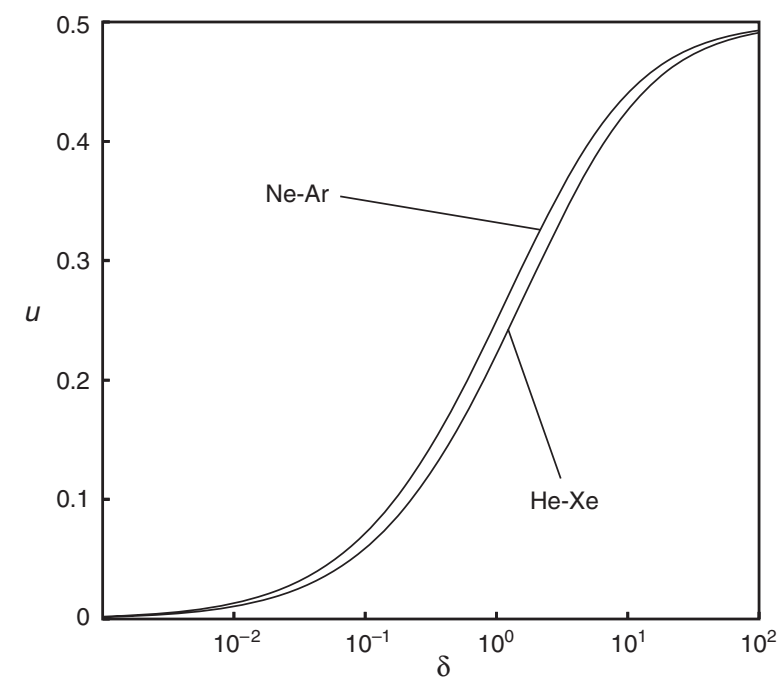

Fig. 3. Mixture velocities at the upper wall of the Couette flow, for $\mathrm{Ne}-\mathrm{Ar}$ and $\mathrm{He}-\mathrm{Xe}(C=0.5)$, in terms of $\delta$.

shown for the $\mathrm{Ne}-\mathrm{Ar}$ and the $\mathrm{He}-\mathrm{Xe}$ mixtures respectively, for $C=0.5$ and $\delta=0.1,1$, and 10. It is seen that for the same $\delta$, the absolute velocities of the $\mathrm{Ne}-\mathrm{Ar}$ mixture are higher than those of the $\mathrm{He}-\mathrm{Xe}$ mixture. In Figure 3, the wall velocities in terms of the rarefaction parameter $\delta$ are plotted for both mixtures with $C=0.5$. It is seen that for all values of $\delta$, the velocity slip at the wall is larger for the He-Xe mixture. The dependence of the results on the molar concentration $C$ is shown in Figure 4 where the wall velocities for the $\mathrm{He}-\mathrm{Xe}$ mixture are plotted for $C=0.1$ and 0.9. It is seen that the maximum difference between the wall velocities for the two different concentrations is about $10 \%$. It is also noted that the corresponding results for the $\mathrm{Ne}-\mathrm{Ar}$ mixture are much closer. It is concluded that for the Couette flow problem the dependence of the results

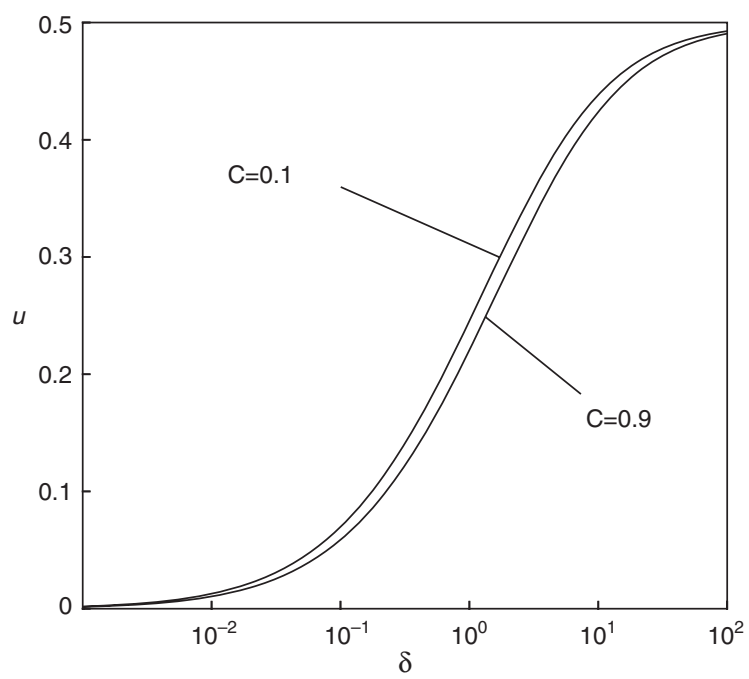

Fig. 4. Mixture velocities at the upper wall of the Couette flow, for $\mathrm{He}-\mathrm{Xe}(C=0.1$ and 0.9$)$, in terms of $\delta$. 

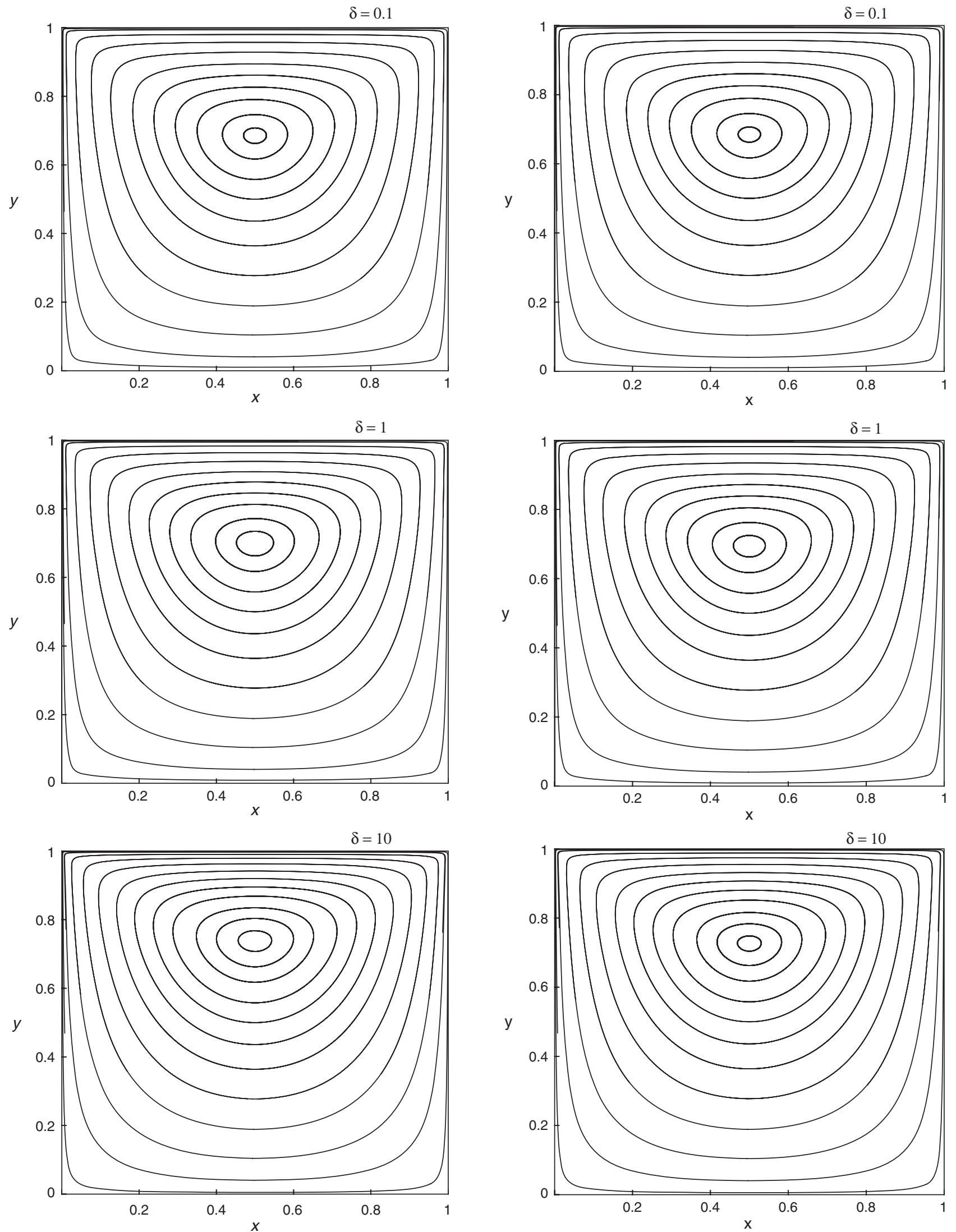

Fig. 5. Streamlines of the cavity flow for $\mathrm{Ne}-\mathrm{Ar}(C=0.5)$ and various values of $\delta$.

Fig. 6. Streamlines of the cavity flow for $\mathrm{He}-\mathrm{Xe}(C=0.5)$ and various values of $\delta$. 

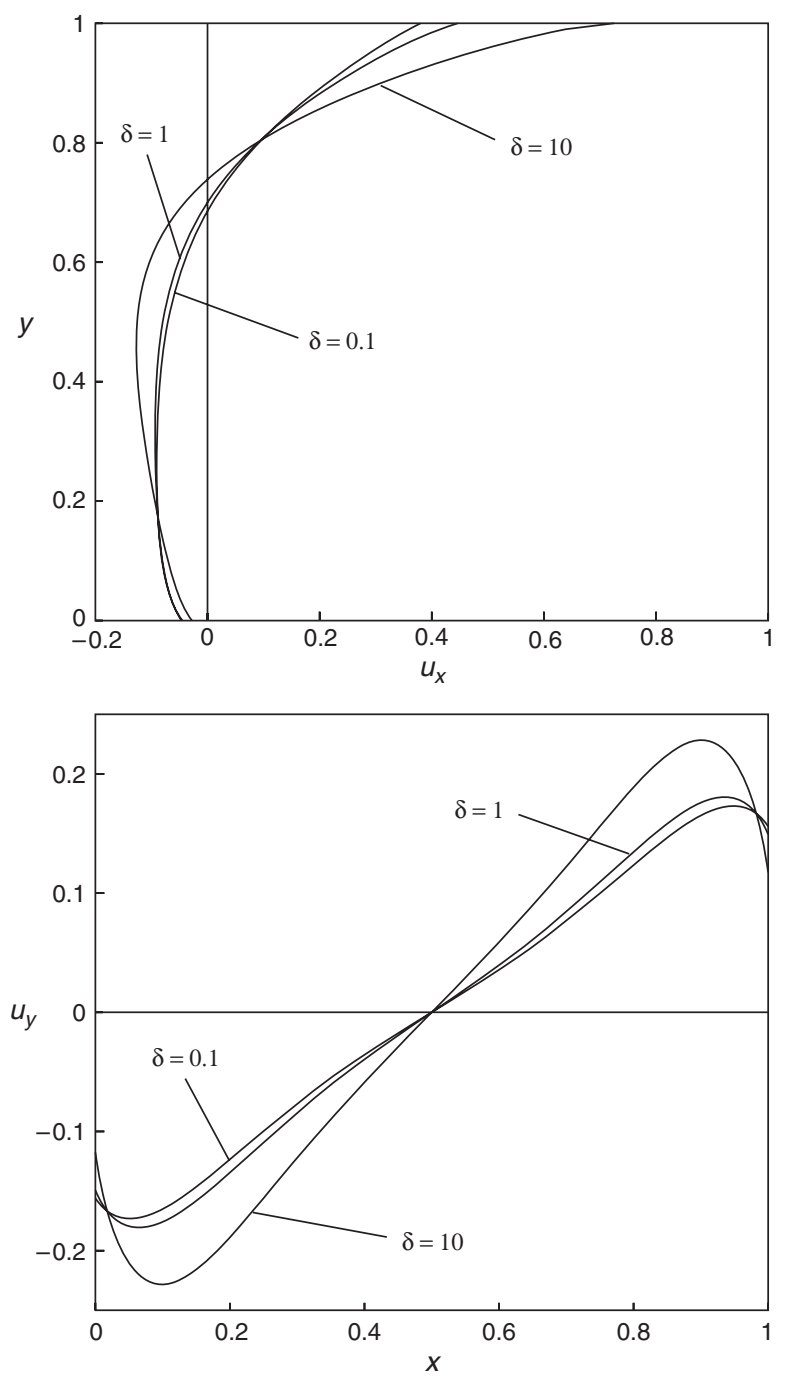

Fig. 7. Velocity profiles at the two cross sections through the center of the vortex for $\mathrm{Ne}-\mathrm{Ar}(C=0.5)$ and various values of $\delta$.

on the molar concentration $C$ is important only for mixtures with particles of different molecular masses. Finally, it is pointed out that the stress in the Couette flow problem depends only on $C$ and $\delta$, and it is independent of the transverse direction $y$. The fact that the stress is constant across the channel is used successfully, as a benchmark, to test the accuracy of the results.

Next, some preliminary results for the square cavity flow problem with $C=0.5$ are presented. In Figures 5 and 6 , the streamlines of the $\mathrm{Ne}-\mathrm{Ar}$ and the $\mathrm{He}-\mathrm{Xe}$ mixtures are plotted for $\delta=0.1,1$, and 10. Although the corresponding flow patterns of the two mixtures are similar, there are differences regarding the location of the vortex center and the corresponding mass flux between the center of the vortex and the moving plate. Velocity components along the vertical and horizontal cross sections passing from the center of the vortex are shown in Figures 7 and 8 for $\delta=0.1,1$, and 10 .
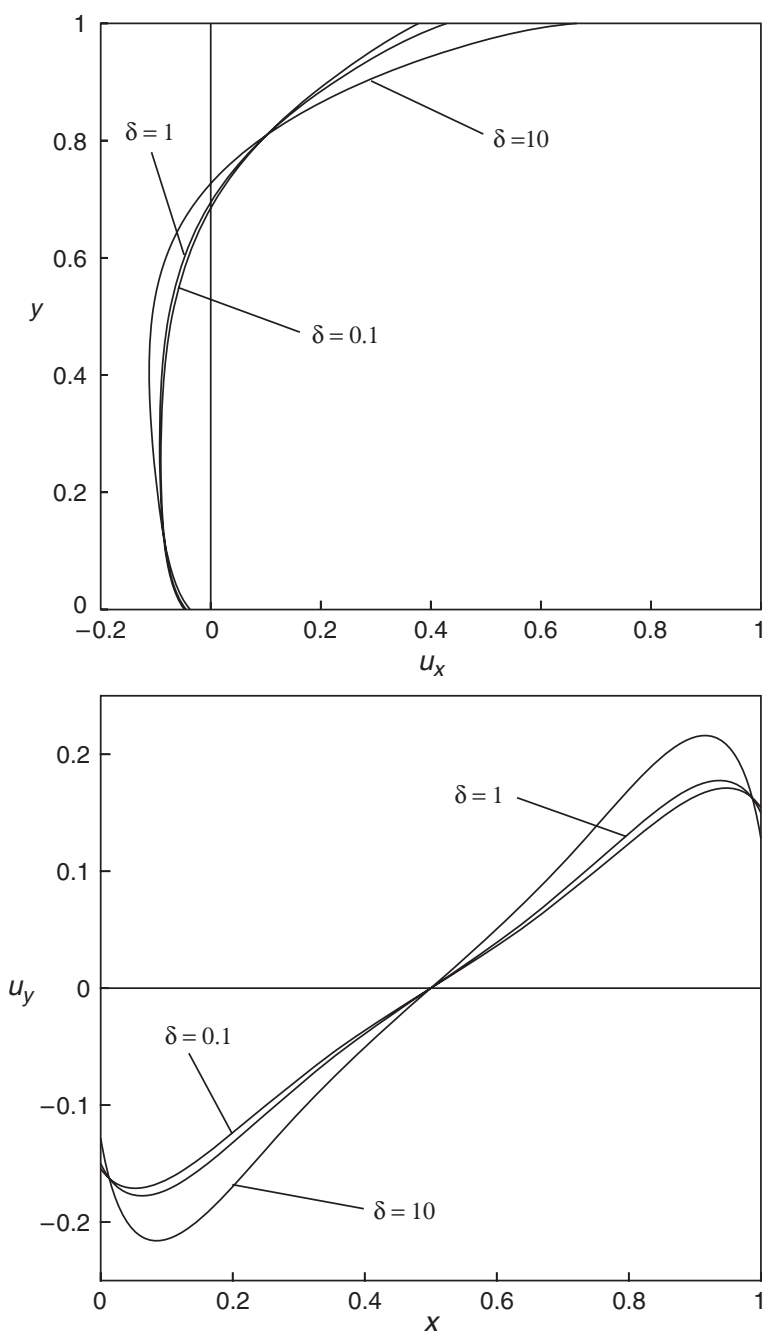

Fig. 8. Velocity profiles at the two cross sections through the center of the vortex for $\mathrm{He}-\mathrm{Xe}(C=0.5)$ and various values of $\delta$.

\section{CONCLUSIONS}

A kinetic-type approach has been applied to solve two typical shear driven micro flows of binary gas mixtures. The solution is complete in the sence that it is accurate for the whole range of rarefaction. Numerical results are presented for two binary mixtures of noble gases for various molar concentrations. It is concluded that gas mixtures can be substituted by a single gas having the mean molecular mass of the mixture, only when the molecular ratio of the species is small. It is demonstrated that the formulation is very efficient and can be implemented as an alternative to classical approaches, such as Navier Stokes solvers with slip boundary conditions.

Acknowledgments: Partial support of this work by the Hellenic Ministry of education through the Program for Basic Research "Hrakleitos" is gratefully acknowledged. 


\section{References and Notes}

1. C. M. Ho and Y. C. Tai, Ann. Rev. Fluid Mech. 30, 579 (1998)

2. G. E. Karniadakis and A. Beskok, Micro Flows, Springer, New York (2001).

3. J. H. Ferziger and H. G. Kaper, Mathematical Theory of Transport Processes in Gases, North-Holland Publishing Company, Amsterdam (1972).

4. C. Cercignani, The Boltzmann Equation and its Applications, Springer, New York (1988).

5. Y. Sone, Theoretical and numerical analysis of the Boltzmann equation-Theory and analysis of rarefied gas flows, In Lecture Notes, Department of Aeronautics and Astronautics, Graduate School of Engineering, Kyoto University.

6. D. Valougeorgis and S. Naris, SIAM J. Sci. Comp. 25, 534 (2003).

7. 3 F. Sharipov and V. Seleznev, J. Phys. Chem. Ref. Data 27, 657 (1998).

8. I. N. Ivchenko, S. K. Loyalka, and R. V. Tompson, J. Vac. Sci. Technol. A 15, 2375 (1997).

9. F. J. McCormack, Phys. Fluids 16, 2095 (1973).

10. F. Sharipov and D. Kalempa, J. Vac. Sci. Technol. A 20, 814 (2002).
11. F. Sharipov and D. Kalempa, Phys. Fluids 15, 1800 (2003).

12. C. E. Siewert and D. Valougeorgis, Eur. J. Mech. B/Fluids 23, 645 (2004).

13. S. Naris, D. Valougeorgis, D. Kalempa, and F. Sharipov, Physica A 336, 294 (2004).

14. S. Naris, D. Valougeorgis, D. Kalempa, and F. Sharipov, Superlattices and Microstructures, 35, 629 (2004).

15. S. Naris, D. Valougeorgis, D. Kalempa, and F. Sharipov, Phys. Fluids 17, 100607 (2005).

16. Y. Onishi, Z. Angew. Math. Phys. (ZAMP), 37, 573 (1986).

17. D. Valougeorgis, Phys. Fluids 31, 521 (1988).

18. C. E. Siewert, J. Quant. Spectr. Rad. Tran. 70, 312 (2001).

19. B. B. Hamel, Phys. Fluids 8, 418 (1965).

20. F. Sharipov, L. M. G. Cumin, and D. Kalempa, Eur. J. Mech. B/Fluids 23, 899 (2004).

21. X. Nie, G. D. Doolen, and S. Chen, J. Stat. Phys. 107, 279 (2002).

22. S. Naris and D. Valougeorgis, Phys. Fluids 17, 097106 (2005).

23. E. E. Lewis and W. F. Miller, Computational Methods in Numerical Transport Theory. Wiley, New York (1984).

24. J. Kestin, K. Knierim, E. A. Mason, B. Najafi, S. T. Ro, and M. Waldman, J. Phys. Chem. Ref. Data 13, 229 (1984). 